\title{
INCORPORATING ECONOMIC POLICY UNCERTAINTY IN US EQUITY PREMIUM MODELS: A NONLINEAR PREDICTABILITY ANALYSIS
}

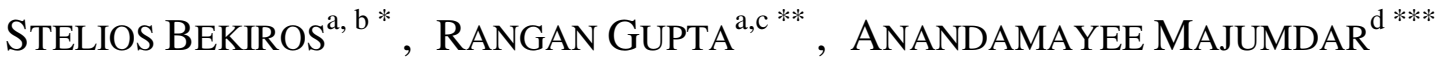 \\ ${ }^{\mathrm{a}} I P A G$ Business School, ${ }^{\mathrm{b}}$ European University Institute (EUI), ${ }^{\mathrm{c}}$ University of Pretoria, \\ ${ }^{\mathrm{d}}$ Soochow University
}

\begin{abstract}
Information on economic policy uncertainty does matter in predicting the US equity premium, especially when accounting for structural instabilities and omitted nonlinearities in their relationship, via a quantile predictive regression approach over the monthly period 1900:1-2014:2. Unlike as suggested by a linear mean-based predictive model, the extended quantile regression model with the incorporation of the EPU proxy, enhances significantly the out-of-sample stock return predictability. This is observed especially when the market is neutral, exhibits a side or mildly upward trending behavior, yet not when the market appears to turn highly bullish.
\end{abstract}

JEL Codes: C22; C53; E60; G10

Keywords: stock markets; economic uncertainty; predictability; quantile regression

*Corresponding author: ${ }^{\text {a } I P A G ~ B u s i n e s s ~ S c h o o l, ~} 184$ Boulevard Saint-Germain, 75006 Paris, France.; Tel.: +33015363 3600 ; Fax: +33014544 4046 ; b Department of Economics, Via della Piazzuola; 43, I-50133, Florence, Italy; Tel.: +39 0554685 916; Fax: +39 0554685 902; E-mail address: stelios.bekiros@eui.eu. ${ }^{* * * c}$ Department of Economics, University of Pretoria, Pretoria, 0002, South Africa; E-mail address: rangan.gupta@up.ac.za.

${ }^{* * * *}$ d Center for Advanced Statistics and Econometrics, Soochow University, Suzhou, China; E-mail address: anandamayee.majumdar@gmail.com. 


\section{INTRODUCTION}

The existing literature on forecasting US stock returns is vast. For practitioners it is of utmost importance to use real-time forecasts in optimal asset allocation, whilst for academics return predictability challenges market efficiency which in turn leads to more realistic asset pricing models (Rapach and Zhou, 2013). However, stock market forecasting is highly controversial as it inherently incorporates stochastic as well as nonlinear components. Understandably a wide array of models e.g., univariate and multivariate, linear and nonlinear ones including various types of predictors namely domestic and international financial, macroeconomic, institutional and behavioural indices, have been recently utilized (Aye et al., 2015). Not surprisingly, the empirical evidence is mixed.

Asset returns are functions of the state variables of the real economy, and the real economy itself displays significant fluctuations. Beyond the standard theoretical justifications of such fluctuations which are mostly based on productivity and/or policy shocks, a recent strand of literature as in Bloom (2009) and Jones and Olson (2013) relates the impact of various forms of policy-generated uncertainty to movements in macroeconomic and financial variables which are expected to affect stock returns. Primarily in-sample empirical evidence in this regard can be found in Antonakakis et al., (2013), Kang and Ratti (2013), Gupta et al.,(2014), Bekiros et al., (2015), Chang et al., (2015) and Jurado et al., (2015). ${ }^{1}$

Against this backdrop, and under the widely held view that predictive models require out-of-sample validation (Rapach and Zhou, 2013), the objective of this paper is to investigate whether the news-based measure of economic policy uncertainty (EPU) introduced by Baker et al. (2013) could help in forecasting the S\&P500-based equity

\footnotetext{
${ }^{1}$ Amongst the papers cited, Gupta et al., (2014) is the only one to analyse out-of-sample forecasting of the US equity premium based on EPU using a linear predictive regression model, but it failed to beat the randomwalk model.
} 
premium. We concentrate on a very broad monthly out-of-sample period (1909:8-2014:2) which encompasses all stock market events in the US over the $20^{\text {th }}$ and the $21^{\text {st }}$ century. Based on the recent contribution by Bekiros and Gupta (2015) who proved the relationship between returns and predictors not being linear, we consider a quantile predictive regression model over and above the standard linear modelling. The quantile-based approach is clearly more informative relative to any linear model, as it investigates the ability of the EPU to forecast the entire conditional distribution of the equity premium, rather than being restricted just to the conditional-mean.

To the best of our knowledge, this is the first attempt to analyse the forecastability of the EPU vis-à-vis the US equity premium, utilizing a quantile regression approach. The rest of the paper is organized as follows: section 2 presents the econometric methodology while section 3 describes the data and discusses the results. Section 4 concludes.

\section{Quantile Predictive Regression model}

The classical mean regression model is given by:

$$
r_{t+1}=\alpha_{i}+\beta_{i} x_{i, t}+\varepsilon_{t+1}
$$

where $r_{t+1}$ is the observed excess return at time $t+1, x_{i, t}$ is a specific regressor / predictor at time $t$, which in our work is EPU and $\varepsilon_{t+1}$ is the error term assumed to be independent with zero mean and variance $\sigma^{2}$. The ordinary least squares (OLS) estimators $\hat{\alpha}_{i}, \hat{\beta}_{i}$ of the parameters in the predictive mean regression model can be estimated by minimizing the quadratic expected loss, $\sum_{t=0}^{T-1}\left(r_{t+1}-\alpha_{i}-\beta_{i} x_{i, t}\right)^{2}$ with respect to $\alpha_{i}, \beta_{i}$. Then, the point forecast of the equity premium at time $t+1$, can be obtained as: $\hat{r}_{i, t+1}=\hat{\alpha}_{i}+\hat{\beta}_{i} x_{i, t}$. 
The aforementioned model specification is primarily devised to predict the mean of $r_{t+1}$, and not its entire distribution. Koenker and Bassett (1978) showed that Quantile Regression estimators are more efficient and robust than mean regression estimators in cases where nonlinearities and deviations from normality exist. Hence, we consider the quantile regression model of the following form:

$$
r_{t+1}=\alpha_{i}^{(\tau)}+\beta_{i}^{(\tau)} x_{i, t}+\varepsilon_{t+1} \quad i=1, \ldots, N,
$$

where $\tau \in(0,1)$ and $\varepsilon_{t+1}$ are assumed independent derived from an error distribution $g_{\tau}(\varepsilon)$ with the $\tau$-th quantile equal to 0 . Model (2) suggests the $\tau$-th quantile of $r_{t+1}$ given $x_{i, t}$, is $Q_{\tau}\left(r_{t+1} \mid x_{i, t}\right)=\alpha_{i}^{(\tau)}+\beta_{i}^{(\tau)} x_{i, t}$, where the intercept and the regression coefficients depend upon $\tau$. The estimators of the parameters of the linear quantile regression model in Eq. (2), $\alpha_{i}{ }^{(\tau)}, \beta_{i}{ }^{(\tau)}$, can be obtained by minimizing the sum $\sum_{t=0}^{T-1} \rho_{\tau}\left(r_{t+1}-\alpha_{i}{ }^{(\tau)}-\beta_{i}{ }^{(\tau)} x_{i, t}\right)$, where $\rho_{\tau}(u)=u(\tau-I(u<0))=\frac{1}{2}[|u|+(2 \tau-1) u]$. The forecast of the $\tau$-th quantile of the distribution of the equity premium at time $t+1$ is obtained as $\hat{r}_{i, t+1}(\tau)=\alpha_{i}^{(\tau)}+$ $\beta_{i}^{(\tau)} x_{i, t}$

\section{EMPIRICAL RESULTS}

The dataset used in the present study covers the monthly period 1900:1-2014:2 and incorporates two variables, namely the US equity premium and the news-based measure of economic policy uncertainty (EPU) introduced by Baker et al. (2013). The equity premium is calculated as the difference of the continuously compounded S\&P 500 returns, including 

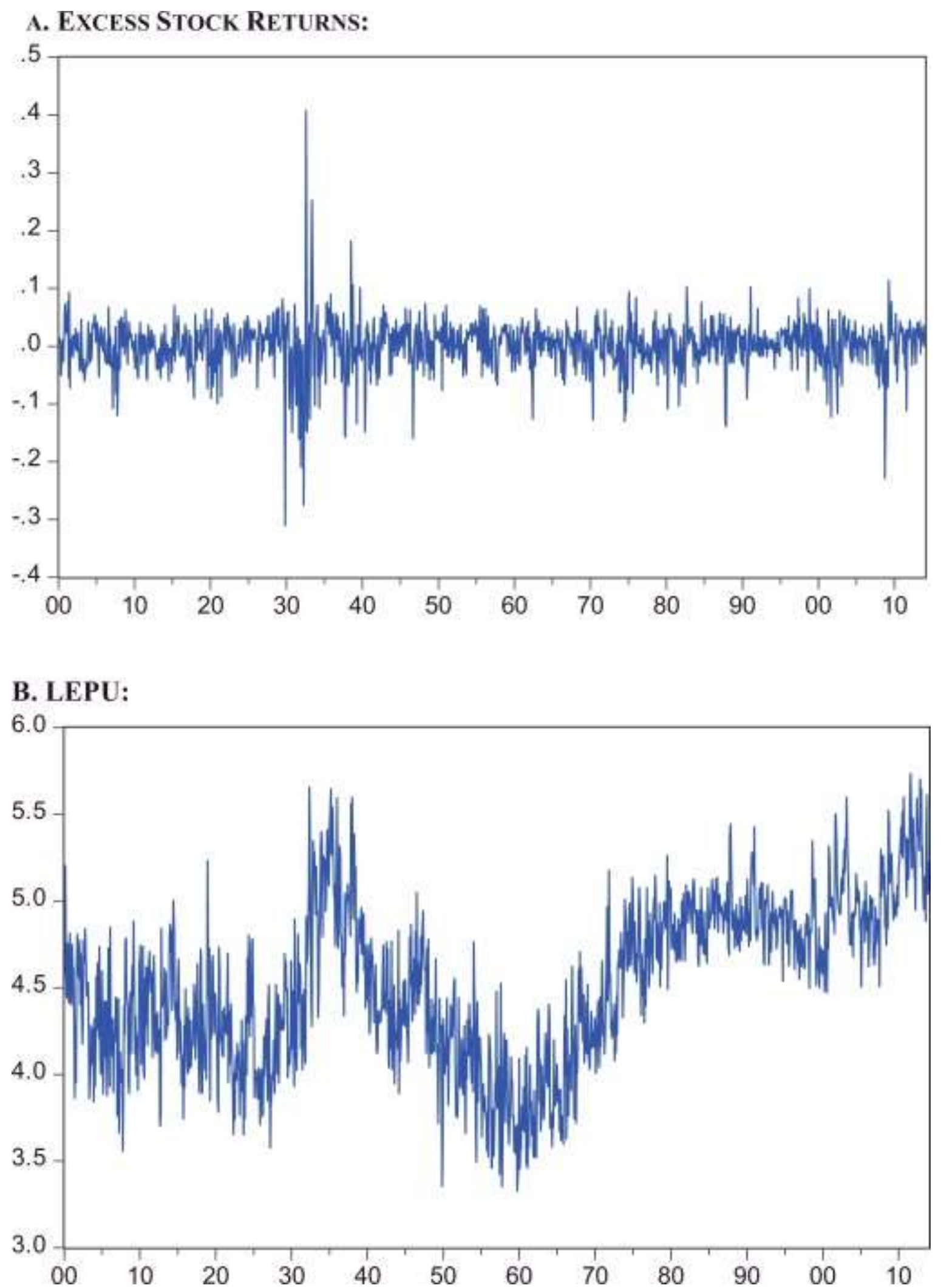

Fig. 1. Data plots of excess returns and natural Logarithm of Economic Policy Uncertainty (LEPU). 
dividends and the three-month Treasury bill rate. ${ }^{2}$ The EPU index is log-transformed ${ }^{3}$ and it is constructed based on month-by-month searches of newspaper articles related to economic and policy uncertainty. ${ }^{4}$ The start and end date of the sample is purely driven by the data availability of the EPU. Fig. 1 plots the equity premium and the natural logarithms of the EPU index.

To determine our in-sample and out-of-sample segmentation with the models in Eq. (1) and Eq. (2) being estimated recursively over the latter, we conduct the Bai and Perron (2003) tests of multiple structural breaks on equation Eq. (1). The test reveals five breaks specifically at 1909:8, 1921:9, 1929:10, 1940:7 and 1966:2, hence our in-sample includes 1900:1-1909:7, while the rest is being utilized as the out-of-sample. ${ }^{5}$ Firstly, when we apply the Jarque-Bera test on the residuals recovered from Eq. (1) the null of normality is overwhelmingly rejected at the highest levels of significance. Furthermore, the Brock et al., (1996, BDS) test when applied on these residuals it also rejects the null of serial dependence at all possible dimensions at all levels of significance, thus provides strong evidence of nonlinearity between the US equity premium and EPU. The results from the structural instability analysis as well as the nonlinearity testing, highlight on the one hand the inappropriateness of the linear predictive regression specification defined in Eq.(1), while on the other hand indicate the necessity to employ a quantile predictive regression, as in Eq. (2). ${ }^{6}$

For the sake of completeness and comparability, we present in Table 1 the forecasting results by the linear predictive regression, aside from the quantile predictive regressions over $\tau=0.05,0.10,0.15 \ldots .0 .95$. The entries in the table report the ratio of the mean square

\footnotetext{
${ }^{2}$ The equity premium until 2013:12, is calculated based on the data available on the website http://www.hec.unil.ch/agoyal/. Beyond this period, data from the FRED database of the Federal Reserve Bank of St. Louis are used.

${ }^{3}$ Standard unit root tests reveal that the natural logarithm of the EPU is stationary. The details of these tests are available upon request from the authors.

${ }^{4}$ Data and further details are available at: http://www.policyuncertainty.com/us_historical.html.

${ }^{5}$ Complete details of the structural break tests are available upon request from the authors.

${ }^{6}$ Complete details of the Jarque-Bera and BDS tests are available upon request by the authors.
} 
forecast errors of Eq. (1) relative the historical average $\hat{r}_{i, t+1}=\hat{\alpha}_{i}$ and the same for Eq. (2) relative to the prevailing quantile model $\hat{r}_{i, t+1}(\tau)=\alpha_{i}{ }^{(\tau)}$. If the ratio score is less than one, then the model with the predictor incorporated outperforms the model without it. It is also important to test whether the superior performance of the model with the EPU - if it holds is statistically different from the appropriate benchmark. Given that one model nests its corresponding benchmark, we use the MSE-F test statistic by McCracken (2007) in order to check whether the outperforming evidence of the ratio being less than one versus the model with uncertainty is statistically significantly.

As it is observed from Table 1 for the linear predictive regression, EPU fails to beat the forecasting performance of the benchmark model. This result was similarly reported by Gupta et al. (2014). However, given the evidence non-normality and nonlinearity, the results of the benchmark linear model cannot be robustly relied upon, hence we move on to the quantile predictive regression model. As we observe from Table 1, the quantile regression model with the included EPU index outperforms the prevailing quantile benchmark significantly at one percent level for $\tau \in[0.05,0.50]$, i.e., around the lower-end to the median of the distribution of the equity premium. ${ }^{7}$ Interestingly though, EPU fails to provide significant forecastability beyond the median.

Overall, unlike as suggested by the linear (mean-based) predictive regression model, the quantile regression model demonstrates that the EPU enhances significantly the out-ofsample predictability especially when the stock market is performing poorly to moderate, yet not when the market appears to turn highly bullish.

\footnotetext{
${ }^{7}$ Qualitatively similar results were obtained when we applied Bayesian versions of the quantile and nonparametric quantile regressions. Complete details are available upon request by the authors.
} 
TABLE 1: MSFE FOR LiNEAR AND QUANTILE PREDICTIVE REgReSSION MODELS

\begin{tabular}{|c|c|}
\hline QQuantile Regression $(\tau)$ & $\bar{c}_{\mathrm{MSFE}_{m} / \mathrm{MSFE}_{b}}$ \\
\hline 0.05 & $0.8964^{\text {**** }}$ \\
\hline 0.10 & $0.8646^{* * *}$ \\
\hline 0.15 & $0.8422^{* * *}$ \\
\hline 0.20 & $0.8858^{* * * *}$ \\
\hline 0.25 & $0.8761^{* * *}$ \\
\hline 0.30 & $0.9186^{* * *}$ \\
\hline 0.35 & $0.9245^{* * *}$ \\
\hline 0.40 & $0.9487^{* * *}$ \\
\hline 0.45 & $0.9703^{* * *}$ \\
\hline 0.50 & $0.9891^{* * *}$ \\
\hline 0.55 & 1.0182 \\
\hline 0.60 & 1.0561 \\
\hline 0.65 & 1.0652 \\
\hline 0.70 & 1.1097 \\
\hline 0.75 & 1.1049 \\
\hline 0.80 & 1.1470 \\
\hline 0.85 & 1.1666 \\
\hline 0.90 & 1.2385 \\
\hline 0.95 & 1.2852 \\
\hline Linear Regression & 1.0030 \\
\hline
\end{tabular}

Note: ${ }^{* * *}$ indicates the $1 \%$ level of significance for the MSE-F statistic, whilst $\tau$ specifies the quantile; MSFE $_{m}$ / $\mathrm{MSFE}_{b}$ signifies the MSFE ratio of the corresponding model over the one generated by the benchmark 


\section{CONCLUSIONS}

The importance of precise stock return forecasts both for practitioners and academics is well-recognized and strongly pursued by market agents. Recent works in the literature provide some conflicting in-sample evidence in favor of the assumption that the economic policy uncertainty index (EPU) possibly drives stock returns.

In an attempt to further substantiate or not this evidence, we compare the forecastability of the US equity premium vis-à-vis the EPU using linear and quantile predictive regression models. The linear regression model with EPU fails to outperform the benchmark model of the historical average of equity premium. However, after suitable testing and thereby accounting for the presence of non-normality and nonlinearity, linear modeling results in misspecification. When we use a quantile predictive regression model, we observe that the economic policy uncertainty index contains significant out-of-sample information around the lower-end to the median of the distribution of the equity premium, albeit not when the market behavior is clearly bullish. 


\section{REFERENCES}

Antonakakis, N., Chatziantoniou, I., and Filis, G. 2013. Dynamic co-movements between stock market returns and policy uncertainty. Economics Letters 120(1), 87-92.

Aye, G. C., Deale, F. W., and Gupta, R. 2015. Does Debt Ceiling and Government Shutdown Help in Forecasting the US Equity Risk Premium? forthcoming in Panoeconomicus

Bai, J., and Perron, P. 2003. Computation and analysis of multiple structural change models. Journal of Applied Econometrics 18(1), 1-22.

Baker, S., Bloom, N., and Davis, S., 2013. Measuring economic policy uncertainty. Chicago Booth Research Paper No.13-02.

Bekiros, S., and Gupta, R. 2015. Predicting stock returns and volatility using consumptionaggregate wealth ratios: A nonlinear approach. Economics Letters 131, 83-85.

Bloom, N. 2009. The impact of uncertainty shocks. Econometrica 77(3), 623-685.

Brock, W. A., Scheinkman, J. A., Dechert, W. D., and LeBaron, B. 1996. A test for independence based on the correlation dimension. Econometric reviews 15(3), 197-235.

Chang, T., Chen, W. Y., Gupta, R.., and Nguyen. D. K. 2015. Are Stock Prices Related to Political Uncertainty Index in OECD Countries? Evidence from Bootstrap Panel Causality Test. forthcoming in Economic Systems.

Gupta, R., Hammoudeh, S., Modise, M. P., and Nguyen, D. K. 2014. Can economic uncertainty, financial stress and consumer sentiments predict US equity premium? Journal of International Financial Markets, Institutions and Money 33, 367-378.

Jones, P. M., and Olson, E. 2013. The time-varying correlation between uncertainty, output, and inflation: Evidence from a DCC-GARCH model. Economics Letters 118(1), 33-37.

Jurado, K., Ludvigson, S. C., and Ng, S. 2015. Measuring Uncertainty. forthcoming in American Economic Review. 
Kang, W., and Ratti, R.A. 2013. Oil shocks, policy uncertainty and stock market returns. Journal of International Financial Markets, Institutions and Money 26(1), 305-318.

Koenker, R., and Bassett Jr, G. 1978. Regression quantiles. Econometrica 46, 33-50.

McCracken, M. W. 2007. Asymptotics for out of sample tests of Granger causality. Journal of Econometrics 140(2), 719-752.

Rapach, D., and Zhou, G. 2013. Forecasting Stock Returns, David E. Rapach and Guofu Zhou: in Handbook of Economic Forecasting, Volume 2A, Graham Elliott and Allan Timmermann (Eds.) Amsterdam: Elsevier , 328-383. 


\section{Appendix A}

Table A1. Summary statistics.

$\begin{array}{cccc} & \text { Excess stock returns } & \text { EPU } & \text { Residual Eq. (1) } \\ \text { Mean } & 0.0012 & 4.5266 & 0.0000 \\ \text { Median } & 0.0048 & 4.5506 & 0.0038 \\ \text { Maximum } & 0.4073 & 5.7349 & 0.4052 \\ \text { Minimum } & -0.3104 & 3.3271 & -0.3100 \\ \text { Std. Dev. } & 0.0431 & 0.4681 & 0.0431 \\ \text { Skewness } & -0.4217 & -0.0829 & -0.4463 \\ \text { Kurtosis } & 14.3285 & 2.3859 & 14.2688 \\ \text { Jarque-Bera } & 7366.4150 & 23.0979 & 7288.9470 \\ \text { p-value } & 0.0000 & 0.0000 & 0.0000 \\ \text { Obs. } & 1370 & 1370 & 1369\end{array}$

Note: EPU denotes economic policy uncertainty in natural logarithms; Eq. (1):

$\mathrm{r}_{\mathrm{t}+1}=\alpha_{\mathrm{i}}+\beta_{\mathrm{i}} \mathrm{x}_{\mathrm{i}, \mathrm{t}}+\varepsilon_{\mathrm{t}+1} \mathrm{rt}+1=\alpha \mathrm{i}+\beta i x \mathrm{i}, \mathrm{t}+\varepsilon \mathrm{t}+1$ Std. Dev. symbolizes the Standard Deviation; $p-$ value corresponds to the test of normality based on the Jarque-Bera test.

Table A2. BDS test.

$\begin{array}{ccccc}\boldsymbol{m} & \boldsymbol{z} \text {-statistic of residual benchmark } & \begin{array}{c}\boldsymbol{p} \text { - } \\ \text { model }\end{array} & \boldsymbol{z} \text {-statistic of residual Eq. } & \begin{array}{c}\boldsymbol{p} \text { - } \\ \text { value }\end{array} \\ \mathbf{2} & 8.7461 & 0.0000 & (\mathbf{1}) & 0.0000 \\ \mathbf{3} & 9.4873 & 0.0000 & 9.6362 & 0.0000 \\ \mathbf{4} & 10.3894 & 0.0000 & 10.2740 & 0.0000 \\ \mathbf{5} & 10.9483 & 0.0000 & 10.9300 & 0.0000 \\ \mathbf{6} & 11.7988 & 0.0000 & 11.7752 & 0.0000\end{array}$

Note: $m$ stands for the embedded dimension; benchmark model:

$\mathrm{r}_{\mathrm{t}+1}=\alpha_{\mathrm{i}}+\varepsilon_{\mathrm{t}+1} \mathrm{rt}+1=\alpha \mathrm{i}+\varepsilon \mathrm{t}+1$; Eq. (1): $\mathrm{r}_{\mathrm{t}+1}=\alpha_{\mathrm{i}}+\beta_{\mathrm{i}} \mathrm{x}_{\mathrm{i}, \mathrm{t}}+\varepsilon_{\mathrm{t}+1} \mathrm{rt}+1=\alpha \mathrm{i}+\beta \mathrm{ixi}, \mathrm{t}+\varepsilon \mathrm{t}+1 ; p$-value corresponds to the test of i.i.d. residuals based on the $z$-statistic of the BDS test. 
Table A3. Bai and Perron (2003) test of multiple structural breaks.

\begin{tabular}{|c|c|c|c|c|}
\hline \multirow[b]{2}{*}{ Breaks } & \multirow[b]{2}{*}{ F-statistic } & Scaled & Weighted & Critical \\
\hline & & F-statistic & F-statistic & Value \\
\hline 1 & 2.7757 & 5.5514 & 5.5514 & 11.02 \\
\hline 2 & 3.1542 & 6.3083 & 6.6334 & 10.48 \\
\hline $3 *$ & 6.1346 & 12.2691 & 14.0693 & 9.61 \\
\hline $4 *$ & 6.2940 & 12.5881 & 15.4305 & 8.99 \\
\hline $5 *$ & 6.0751 & 12.1502 & 15.7524 & 8.50 \\
\hline Max stat & & 12.5881 & UDMax criti & 1 value** \\
\hline Max sta & & 15.7524 & WDMax crit & al value** \\
\hline
\end{tabular}

Sequential F-stat. determined breaks: 0

Significant F-stat. largest breaks: $\quad 5$

UDmax determined breaks: 4

WDmax determined breaks: 5

Estimated break dates:

1: 1942:05

2: 1929:10, 1940:07

3: 1921:09, 1929:10, 1940:07

4: 1909:08, 1921:09, 1929:10, 1940:07

5: 1909:08, 1921:09, 1929:10, 1940:07, 1966:02

Note: The Bai-Perron tests is presented for 1 to $M$ globally determined breaks. The sample spans 1900M01-2014M02. The investigated EPU breakpoint model includes the first lag of EPU (i.e., EPU(-1)) and a constant term C. We allow for heterogeneous error distributions across breaks; * denotes significance at the 0.10 level, while ${ }^{* *}$ denotes the Bai-Perron critical values (Econometric Journal, 2003). 